ScIDice

\section{Awareness Of Biomedical Applications Of Reactive Oxygen Species Generation By Titanium Nano- particles Among Dental Students}

\section{International Journal of Dentistry and Oral Science (IJDOS) \\ ISSN: $2377-8075$}

Research Article

Dhanraj Ganapathy ${ }^{1 *}$, Martina Catherine ${ }^{2}$

${ }^{1}$ Professor \& Head of Department, Department of Prosthodontics, Saveetha Dental College and Hospitals, Saveetha Institute of Medical and Technical Sciences, Chennai, India.

${ }^{2}$ Tutor, Department of Prosthodontics, Saveetha Dental College and Hospitals, Saveetha Institute of Medical and Technical Sciences, Chennai, India.

\title{
Abstract
}

Introduction: Some NPs, once released into the body through different internalization methods such as oral, parenteral, inhalation administration and skin adsorption, can affect redox homeostasis both by generating ROS or lessening scavenging pathways. Titanium dioxide (TiO2) is one of the most extensively used nanomaterials for several applications, but the photocatalytic properties of TiO2NPs have raised many issues as a result of ROS generation while UV irradiation is performed. Aim: This survey was conducted for assessing the awareness of Biomedical Applications of Reactive Oxygen Species Generation by Titanium Nanoparticles among dental students.

Materials and Method: A cross-section research was conducted with a self-administered questionnaire containing ten questions distributed amongst 100 dental students. The questionnaire assessed the awareness about Reactive Oxygen Species Generation by Titanium Nanoparticles, their photocatalytic properties, anti-cancer properties, prooxidant effects, mechanism of action and toxicity effects, The responses were recorded and analysed.

Results: $10 \%$ of the respondents were aware of the Biomedical Applications of Reactive Oxygen Species Generation by Titanium Nanoparticles. $9 \%$ were aware of photocatalytic properties of Titanium Nanoparticle, $9 \%, 5 \%$ were aware of anticancer activities of Titanium Nanoparticles, $5 \%$ were aware of prooxidant effects of Titanium Nanoparticles and, $5 \%$ were aware of mechanism of action and toxicity effects, of Titanium Nanoparticles.

Conclusion: There is limited awareness amongst dental students about Biomedical Applications of Reactive Oxygen Species Generation by Titanium Nanoparticles. Enhanced awareness initiatives and dental educational programmes together with increased importance for curriculum improvements that further promote knowledge and awareness of Titanium Nanoparticles therapy.

Keywords: Awareness; Titanium; Nanoparticles; Students; Medicinal; Reactive Oxygen Species.

\section{Introduction}

Nanoscience refers to the study and application of tiny materials with dimensions equal to or less than $100 \mathrm{~nm}$ of which many other fields, such as material science, engineering, physics, chemistry, biology and medicine, can take advantage. One of the most active areas of research in this field is the study and the development of nanostructured materials (NSMs) and nanoparticles (NPs) [1] NSMs and NPs have unique tunable physicochemical features such as catalytic activity, electrical and thermal conductivity, light absorption and scattering that, starting from bulk counterparts, allow enhanced performance to be exploited by many different areas such as food industry, agriculture, cosmetics and, of course, medicine. In the latter area, NSMs and NPs have found suitable applications in fluorescent biological labeling, pathogen detection, protein analysis, DNA structure probing, tissue engineering, separation and purification of cells and biological molecules magnetic resonance imaging (MRI) contrast enhancement, drug and gene delivery [2].

Particularly, in more recent decades, NPs have been successfully used in the clinic as effective tools for alternative therapy such as

\footnotetext{
*Corresponding Author:

Dhanraj Ganapathy,

Professor \& Head of Department, Department of Prosthodontics, Saveetha Dental College and Hospitals, Saveetha Institute of Medical and Technical Sciences, Chennai, India. Tel: 9841504523

E-mail: dhanrajmganapathy@yahoo.co.in
}

Received: September 12, 2021

Accepted: September 20, 2021

Published: September 21, 2021

Citation: Dhanraj Ganapathy, Martina Catherine. Awareness Of Biomedical Applications Of Reactive Oxygen Species Generation By Titanium Nanoparticles Among Dental Students. Int J Dentistry Oral Sci. 2021;8(9):4363-4366. doi: http://dx.doi.org/10.19070/2377-8075-21000888

Copyright: Dhanraj Ganapathy ${ }^{\circ} 2021$. This is an open-access article distributed under the terms of the Creative Commons Attribution License, which permits unrestricted use, distribution and reproduction in any medium, provided the original author and source are credited. 
photodynamic therapy (PDT), high-intensity focused ultrasound therapy (HIFU), photothermal therapy (PPT) and sonodynamic therapy [3]. The ever-increasing success of these therapies is due to their ability to induce the death of prokaryotic and eukaryotic cells through key cellular mechanisms such as that of induction of NP-mediated reactive oxygen species (ROS) generation [22]. Some NPs, once released into the body through different internalization methods such as oral, parenteral, inhalation administration and skin adsorption, can affect redox homeostasis both by generating ROS or lessening scavenging pathways [4].

Titanium dioxide ( $\mathrm{TiO} 2)$ is one of the most extensively used nanomaterials for several applications, but the photocatalytic properties of TiO2NPs have raised many issues as a result of ROS generation while UV irradiation is performed. Indeed, electrons in the $\mathrm{TiO} 2$ valence band absorb the photon energy under UVA irradiation, and jump to the conduction band, allowing extraction of electrons from water or hydroxyl ions generating hydroxyl radicals by valence band holes. Other methods of ROS formation, such as superoxide anion and singlet oxygen by additionally mechanisms, have also been demonstrated [5].

The photocatalytic properties of this NP make TiO2 a valuable competitor for some biomedical applications, such as in killing microorganisms and treating malignant tumors. From this initial investigation, other researchers have studied the cytotoxicity by photoexcited TiO2 on cancer cells, but more intriguing has been two recent scientific works where the TiO2NPs have been found to be effective in PT'T against a melanoma cancer model and also as sonosensitizer in SDT against a breast cancer model [6].

NPs of varying chemical composition such as metal oxides have been shown to induce oxidative stress and, in this regard, NPs have been reported to influence intracellular calcium concentrations, activate transcription factors and modulate cytokine production via generation of free radicals [7]. The main key factors involved in NP-induced ROS include: prooxidant functional groups on the reactive surface of NPs, active redox cycling on the surface of NPs due to transition metal-based NPs (MNPs), and particle-cell interactions. With regards to these key factors, several studies have shown the significance of reactive particle surfaces in ROS generation [8]. Our research experience has prompted us in pursuing this research [9-20]. This survey was conducted for assessing the awareness of Biomedical Applications of Reactive Oxygen Species Generation by Titanium Nanoparticles among dental students.

\section{Materials and Methods}

A cross-section research was conducted with a self-administered questionnaire containing ten questions distributed amongst 100 dental students. The questionnaire assessed the awareness about Reactive Oxygen Species Generation by Titanium Nanoparticles, their photocatalytic properties, anti-cancer properties, prooxidant effects, mechanism of action and toxicity effects, the responses were recorded and analysed.

\section{Results}

$10 \%$ of the respondents were aware of the Biomedical Applications of Reactive Oxygen Species Generation by Titanium Nanoparticles (Fig 1). $9 \%$ were aware of photocatalytic properties of Titanium Nanoparticle (Fig 2), 5\% were aware of anti-cancer activities of Titanium Nanoparticles (Fig 3), 5\% were aware of prooxidant effects of Titanium Nanoparticles (Fig 4) and, 5\% were aware of mechanism of action and toxicity effects, of Titanium Nanoparticles (Fig 5).

\section{Discussion}

Reactive oxygen species, key signaling molecules during cell signaling and homeostasis, are produced in cells by oxidases, originating from the excitation and univalent reduction of the molecular oxygen, which leads to the generation of hydroxyl radicals, superoxide anion and hydrogen peroxide [21]. Briefly, molecular oxygen generates superoxide anion, the primary ROS, via reduction of one electron catalyzed by nicotinamide adenine dinucleo-

Figure 1. Awareness of the Biomedical Applications of Reactive Oxygen Species Generation by Titanium Nanoparticles.

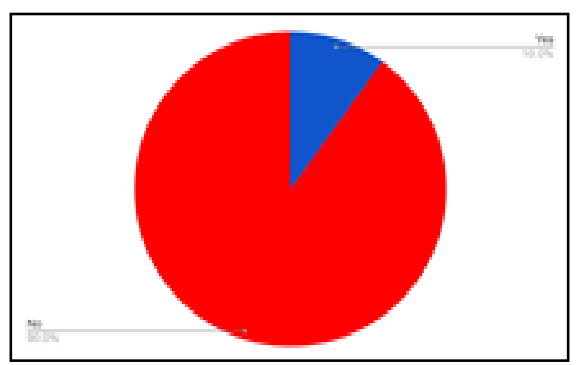

Figure 2. Awareness of the photocatalytic properties of Titanium Nanoparticle.

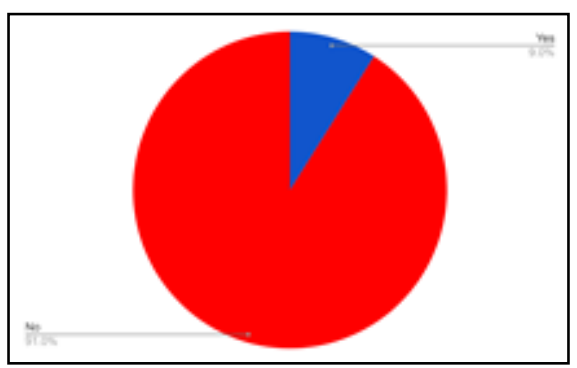


Figure 3. Awareness of the anti-cancer activities of Titanium Nanoparticles.

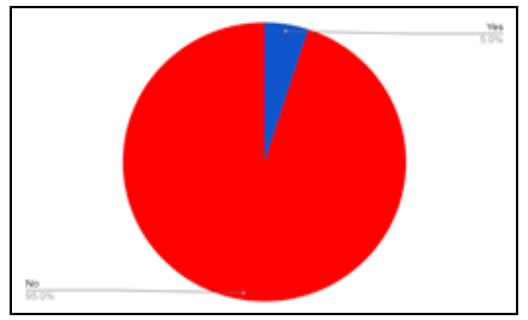

Figure 4. Awareness of the prooxidant effects of Titanium Nanoparticles.

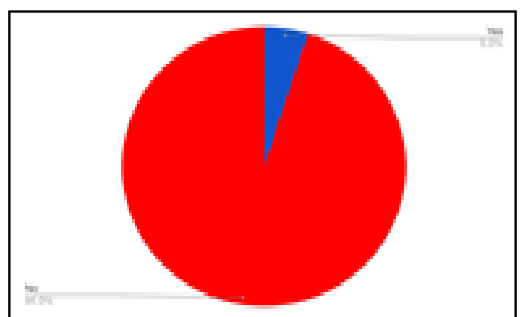

Figure 5. Awareness of the mechanism of action and toxicity effects, of Titanium Nanoparticles

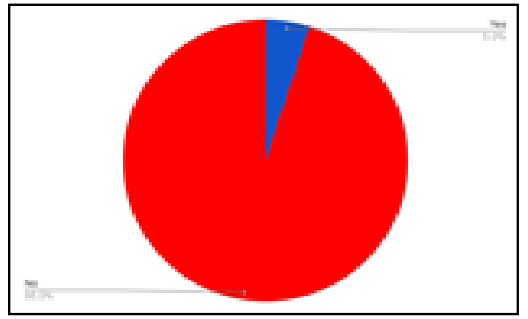

tide phosphate (NADPH) oxidase. Further reduction of oxygen may either lead to hydrogen peroxide or hydroxyl radicals via dismutation and metal-catalyzed Fenton reaction, respectively [22]. Some of the endogenous sources of ROS include mitochondrial respiration, inflammatory response, microsomes and peroxisomes. However, the occurrence of free radicals from essential byproducts of mitochondrial respiration and transition metal ioncatalyzed Fenton-type reactions mainly can regulate many signal transduction paths in a dose-dependent way. While low or medium ROS levels raise mitogenic signaling via reversible oxidations, high ROS levels lead to nucleic acids and lipid oxidation and peroxidation, resulting in cellular apoptosis and necrosis phenomena [23].

The photocatalytic properties of this NP make TiO2 a valuable competitor for some biomedical applications, such as in killing microorganisms and treating malignant tumors [24]. The latter application has been investigated since 1992, when Sakai et al. studied the effect of photoexcited TiO2 on cancer cells in in vitro studies [25]. From this initial investigation, other researchers have studied the cytotoxicity by photoexcited $\mathrm{TiO} 2$ on cancer cells [26], but more intriguing has been two recent scientific works where the TiO2NPs have been found to be effective in PTT against a melanoma cancer model and also as sonosensitizer in SDT against a breast cancer model [6].

The investigators assessed the application of PEGylated TiO2NPs in inducing hyperthermia and necrosis in in vivo melanoma tumors after PTT consisting of a continuous wave near-infrared (NIR) laser diode at $808 \mathrm{~nm}$ wavelength with an intensity of 2 $\mathrm{W} / \mathrm{cm} 2$ for seven minutes. Four mice groups were enrolled in the experiments and the main result showed that in the PEGylated TiO2NPs + laser therapy group, not only did the tumor growth cease, but the tumor size also shrank according to the ultrasonography images and the histopathological examination in the three days following the experiment. Interestingly, five mice from the PEGylated TiO2NPs + laser therapy group were euthanized after three months of follow-up to demonstrate biocompatibility of these PEGylated TiO2NPs. However, no data about the survival rate of those animals were reported.

The latter work investigated TiO2NPs, more precisely the spherical carbon-doped titanium dioxide nanoparticles (C-doped TiO2NPs), as a sonosensitizer in SDT in order to overcome the major limitation associated with cancer therapies that involve electromagnetic waves, i.e., the shallow penetration depth of light sources into tumor tissue [27]. Taking this into consideration, Yang and colleagues investigated whether C-doped TiO2NPs were able to suppress the proliferation of 4T1 breast cancer cell line in both in vitro and in vivo models in combination with US treatment (US frequency of $1.0 \mathrm{MHz}$ and a duty cycle of $50 \%$ with a negative pressure of $0.33 \mathrm{MPa}$ and intensity of $1.8 \mathrm{~W} / \mathrm{cm} 2$ for $90 \mathrm{~s}$ ) in order to inhibit tumor growth.

Firstly, in the in vitro study, the authors quantified ROS production between treatment groups and found that C-doped TiO2NPs, in combination with US, significantly increased the level of ROS compared to control group. This result corroborated that, under US irradiation, ROS generation could be improved in the presence of C-doped TiO2NPs. Thereafter SDT cytotoxicity was evaluated confirming that SDT, i.e., 4T1 cells cultured with C-doped TiO2NPs and subjected to US exposure, induced higher cytotoxicity in 4T1 cells than the other treatment groups. Interesting was the speculation about the possible pathway of cell damage induced by SDT, where the authors suggested a role of sonoluminescence in the C-doped TiO2NPs activation to gener- 
ate more ROS and kill 4T1 breast cancer cells [28].

Finally, in this work, to further investigate the cell death of 4T1 cells induced by SDT, an in vivo study was performed. Groups of 5 nude mice were enrolled, all bearing subcutaneous 4TI breast cancer cells, and the data showed that the C-doped TiO2NPs group $(150 \mathrm{mg} / \mathrm{mL}$ C-doped TiO2NPs at day 0 and day 7$)$ and US group (PBS at day 0 and day 7) could not suppress the tumor growth, while the SDT group $(150 \mathrm{mg} / \mathrm{mL}$ C-doped TiO2NPs at day 0 and day 7 with US exposure) was able to significantly delay tumor growth in that the relative tumor volume at endpoint was almost half that of other control groups. Moreover, by using histologic staining of the tumor site, authors observed that SDT enhanced the ability to cause 4T1 cell death compared to the other groups, confirming that C-doped TiO2NPs could be considered as sonosensitizers for sonodynamic treatments, and in general as an efficient strategy for alternative cancer treatments.

In addition to the prooxidant effect of NPs, ROS are also induced endogenously where the mitochondrion is a major cell target for NP-induced oxidative stress. Specifically, once NPs gain access into the mitochondria, they stimulate ROS via impaired electron transport chain, structural damage, activation of NADPH-like enzyme systems and depolarization of the mitochondrial membrane $[29,30]$.

Despite these promising results, MNPs' potential drug ability requires further extensive evaluation before they can reach clinical applications. Therefore, future research involving MNPs should consist of robust clinical studies with a predominant focus on acceleration of their clinical translation for biomedical uses.

\section{Conclusion}

There is limited awareness amongst dental students about Biomedical Applications of Reactive Oxygen Species Generation by Titanium Nanoparticles. Enhanced awareness initiatives and dental educational programmes together with increased importance for curriculum improvements that further promote knowledge and awareness of Titanium Nanoparticles therapy.

\section{References}

[1]. Jeevanandam J, Barhoum A, Chan YS, Dufresne A, Danquah MK. Review on nanoparticles and nanostructured materials: history, sources, toxicity and regulations. Beilstein J Nanotechnol. 2018 Apr 3;9:1050-1074. Pubmed PMID: 29719757.

[2]. Jin S, Ye K. Nanoparticle-mediated drug delivery and gene therapy. Biotechnol Prog. 2007 Jan-Feb;23(1):32-41. Pubmed PMID: 17269667.

[3]. Chan WC, editor. Bio-applications of Nanoparticles. Springer Science \& Business Media; 2009 Sep 29.

[4]. Abdal Dayem A, Hossain MK, Lee SB, Kim K, Saha SK, Yang GM, Choi HY, Cho SG. The Role of Reactive Oxygen Species (ROS) in the Biological Activities of Metallic Nanoparticles. Int J Mol Sci. 2017 Jan 10;18(1):120. Pubmed PMID: 28075405.

[5]. Hirakawa K, Hirano T. Singlet oxygen generation photocatalyzed by TiO2 particles and its contribution to biomolecule damage. Chemistry Letters. 2006 Aug;35(8):832-3.

[6]. Behnam MA, Emami F, Sobhani Z, Dehghanian AR. The application of titanium dioxide $(\mathrm{TiO} 2)$ nanoparticles in the photo-thermal therapy of melanoma cancer model. Iran J Basic Med Sci. 2018 Nov;21(11):1133-1139. Pubmed PMID: 30483386

[7]. Chakraborti S, Chakraborti T, Das SK, Chattopadhyay D. Oxidative Stress in Lung Diseases: Volume 1. Springer Nature; 2019. 491 p.

[8]. Schins RP. Mechanisms of genotoxicity of particles and fibers. Inhal Toxicol. 2002 Jan;14(1):57-78. Pubmed PMID: 12122560
[9]. Hemalatha R, Ganapathy D. Disinfection of Dental Impression- A Current Overview. Journal of Pharmaceutical Sciences and Research. 2016 Jul;8(7):661-4.

[10]. Ramya G, Pandurangan K, Ganapathy D. Correlation between anterior crowding and bruxism-related parafunctional habits. Drug Invention Today. 2019 Oct 15;12(10).

[11]. Anjum AS, Ganapathy D, Kumar K. Knowledge of the awareness of dentists on the management of burn injuries on the face. Drug Invention Today. 2019 Sep $1 ; 11(9)$.

[12]. Inchara R, Ganapathy D, Kumar PK. Preference of antibiotics in pediatric dentistry. Drug Invent Today. 2019 Jun 15;11:1495-8.

[13]. Philip JM, Ganapathy DM, Ariga P. Comparative evaluation of tensile bond strength of a polyvinyl acetate-based resilient liner following various denture base surface pre-treatment methods and immersion in artificial salivary medium: An in vitro study. Contemp Clin Dent. 2012 Jul;3(3):298-301. Pubmed PMID: 23293485.

[14]. Gupta A, Dhanraj M, Sivagami G. Implant surface modification: review of literature. The Internet Journal of Dental Science. 2009;7(1):10.

[15]. Indhulekha V, Ganapathy D, Jain AR. Knowledge and awareness on biomedical waste management among students of four dental colleges in Chennai, India. Drug Invention Today. 2018 Dec 1;10(12):32-41.

[16]. Mohamed Usman JA, Ayappan A, Ganapathy D, Nasir NN. Oromaxillary prosthetic rehabilitation of a maxillectomy patient using a magnet retained two-piece hollow bulb definitive obturator; a clinical report. Case Rep Dent. 2013;2013:190180. Pubmed PMID: 23533823.

[17]. Ganapathy DM, Joseph S, Ariga P, Selvaraj A. Evaluation of the influence of blood glucose level on oral candidal colonization in complete denture wearers with Type-II Diabetes Mellitus: An in vivo Study. Dent Res J (Isfahan). 2013 Jan;10(1):87-92. Pubmed PMID: 23878569.

[18]. Menon A, Ganapathy DM, Mallikarjuna AV. Factors that influence the colour stability of composite resins. Drug Invention Today. 2019 Mar 1;11(3).

[19]. Dhanraj G, Rajeshkumar S. Anticariogenic Effect of Selenium Nanoparticles Synthesized Using Brassica oleracea. Journal of Nanomaterials. $2021 \mathrm{Jul}$ $10 ; 2021$.

[20]. Ganapathy D, Department of Prostodontics, Saveetha Dental College, Saveetha Institute of Medical and Technical Sciences, - C, India. Nanobiotechnology in combating CoVid-19 [Internet]. Vol. 16, Bioinformation. 2020. p. 828-30. Available from:

[21]. Manke A, Wang L, Rojanasakul Y. Mechanisms of nanoparticle-induced oxidative stress and toxicity. Biomed Res Int. 2013;2013:942916. Pubmed PMID: 24027766.

[22]. Thannickal VJ, Fanburg BL. Reactive oxygen species in cell signaling. American Journal of Physiology-Lung Cellular and Molecular Physiology. 2000 Dec 1;279(6):L1005-28.

[23]. Snezhkina AV, Kudryavtseva AV, Kardymon OL, Savvateeva MV, Melnikova NV, Krasnov GS, Dmitriev AA. ROS Generation and Antioxidant Defense Systems in Normal and Malignant Cells. Oxid Med Cell Longev. 2019 Aug 5;2019:6175804. Pubmed PMID: 31467634.

[24]. Foster HA, Ditta IB, Varghese S, Steele A. Photocatalytic disinfection using titanium dioxide: spectrum and mechanism of antimicrobial activity. Appl Microbiol Biotechnol. 2011 Jun;90(6):1847-68. Pubmed PMID: 21523480.

[25]. Sakai H, Ito E, Cai RX, Yoshioka T, Kubota Y, Hashimoto K, Fujishima A. Intracellular $\mathrm{Ca} 2+$ concentration change of $\mathrm{T} 24$ cell under irradiation in the presence of TiO2 ultrafine particles. Biochim Biophys Acta. 1994 Nov 11;1201(2):259-65. Pubmed PMID: 7947940.

[26]. Kubota Y, Shuin T, Kawasaki C, Hosaka M, Kitamura H, Cai R, Sakai H, Hashimoto K, Fujishima A. Photokilling of T-24 human bladder cancer cells with titanium dioxide. Br J Cancer. 1994 Dec;70(6):1107-11. Pubmed PMID: 7981061.

[27]. Yang CC, Wang CX, Kuan CY, Chi CY, Chen CY, Lin YY, Chen GS, Hou $\mathrm{CH}$, Lin FH. Using C-doped TiO2 Nanoparticles as a Novel Sonosensitizer for Cancer Treatment. Antioxidants (Basel). 2020 Sep 17;9(9):880. Pubmed PMID: 32957611.

[28]. Canaparo R, Foglietta F, Giuntini F, Francovich A, Serpe L. The bright side of sound: perspectives on the biomedical application of sonoluminescence. Photochem Photobiol Sci. 2020 Sep 9;19(9):1114-1121. Pubmed PMID: 32685951.

[29]. Sioutas C, Delfino RJ, Singh M. Exposure assessment for atmospheric ultrafine particles (UFPs) and implications in epidemiologic research. Environ Health Perspect. 2005 Aug;113(8):947-55. Pubmed PMID: 16079062.

[30]. Xia T, Kovochich M, Brant J, Hotze M, Sempf J, Oberley T, Sioutas C, Yeh JI, Wiesner MR, Nel AE. Comparison of the abilities of ambient and manufactured nanoparticles to induce cellular toxicity according to an oxidative stress paradigm. Nano Lett. 2006 Aug;6(8):1794-807. Pubmed PMID: 16895376. 